\title{
A Very Rare Case of Coronary Artery Bypass Grafting in a Progeria Child
}

\author{
Rui Pedro Soares Cerejo, MD' $\oplus$, Rui A. N. Rodrigues, MD', \\ José D. Martins, MD ${ }^{2}$, Carolina G. E. C. Torres, MD $^{3}$, Lídia M. Sousa, MD ${ }^{4}$, \\ Fátima F. Pinto, $M D, P^{2}{ }^{2}$, and José I. G. Fragata, $M D, P^{\prime}{ }^{\prime}$
}

\begin{abstract}
Hutchinson-Gilford progeria syndrome is a rare genetic disorder, characterized by progressive premature aging and early death in the first or second decade of life, usually secondary to cardiovascular events (myocardial infarction and stroke). We report a case of a l4-year-old boy with progeria syndrome and cardiac arrest due to myocardial infarction, who was submitted to an immediate coronary angiography which revealed left main stem and three-vessel coronary artery disease. A prompt double bypass coronary artery grafting surgery was performed, and, despite successful coronary reperfusion, the patient remained in coma and brain death was declared on fourth day after surgery.
\end{abstract}

\section{Keywords}

Hutchinson-Gilford, progeria, coronary artery disease, myocardial infarction, pediatric, cardiac catheterization/intervention, coronary artery surgery, off-pump surgery

Submitted May 21, 2018; Accepted September 02, 2018.

\section{Introduction}

Hutchinson-Gilford progeria syndrome (HGPS) was first described in 1886, and since then, around 100 cases have been reported. ${ }^{1}$ It is a rare autosomal dominant genetic disorder (1 per 4,000,000 live births). ${ }^{2}$ Mutation in exon 11 of the gene coding for Lamin A (LMNA-gene) generates an alternatively spliced form of Lamin A, termed progerin. The accumulation of this deletion mutant Lamin A, causes nuclear instability and changes in chromatin and gene expression and is thought to be responsible for HGPS. ${ }^{3,4}$

Progeria is characterized by a general appearance of accelerated aging. The birth of a child with progeria is generally uneventful, but development is characterized by a delayed growth. Dysmorphic features, such as retarded growth, baldness, macrocephaly, skin changes, and osteoarticular malformations, appear with age. Accelerated atherosclerosis of carotid and coronary arteries causes stroke and myocardial infarction, which are the most common cause of death in this population, with mean survival reported of 12.6 years. ${ }^{4}$ Because of their sudden and rare occurrence, there are scarce descriptions in the literature of acute interventional or surgical treatment of cardiac events in patients with HGPS. ${ }^{4}$ To our knowledge, there is only one report of a successful percutaneous coronary intervention in this setting, and there are very few reports of a coronary surgery in these patients. ${ }^{5}$

\section{Clinical Summary}

We present a case of a 14-year-old boy with a diagnosis of HGPS and typical features of this syndrome: failure to thrive (17 kg), osteopenia, baldness, skin changes, and skeletal and articular malformations. He had a cardiovascular history of left internal carotid stenosis, middle cerebral artery ostial lesions, and a hemorrhagic stroke submitted to a decompressive craniotomy at age of eight. He had also a previous medical history of hypercholesterolemia, right eye keratitis, and multiple orthopedic and general surgeries. He was under acetylsalicylic acid and lonafarnib. There was no history of any previous cardiovascular procedure.

\footnotetext{
' Cardiothoracic Surgery Department, Hospital de Santa Marta-Centro Hospitalar Lisboa Central, Lisbon, Portugal

${ }^{2}$ Pediatric Cardiology Department, Hospital de Santa Marta-Centro Hospitalar Lisboa Central, Lisbon, Portugal

${ }^{3}$ Thoracic Surgery Department, Hospital Pulido Valente-Centro Hospitalar Lisboa Norte, Lisbon, Portugal

${ }^{4}$ Cardiology Department, Hospital de Santa Marta-Centro Hospitalar Lisboa Central, Lisbon, Portugal
}

Corresponding Author:

Rui Pedro Soares Cerejo, Cardiothoracic Surgery Department, Hospital de Santa Marta, Rua de Santa Marta n50, I I69-024 Lisbon, Portugal.

Email: ruipscerejo@gmail.com 


\begin{tabular}{|ll|}
\hline \multicolumn{2}{|l|}{ Abbreviations } \\
CABG & coronary artery bypass grafting \\
CPB & cardiopulmonary bypass \\
HGPS & Hutchinson-Gilford progeria syndrome \\
ICU & intensive care unit \\
ITA & internal thoracic artery \\
LAD & left anterior descending artery \\
LITA & left internal thoracic artery \\
RITA & right internal thoracic artery \\
\hline
\end{tabular}

Five hours after an orthopedic surgery (right hip and left femur intervention) in another hospital, when recovering in a pediatric surgery ward, he suddenly developed chest pain, sweating, vomiting, and fainting followed by cardiac arrest. Advanced cardiac life support was started and continued allegedly for four minutes when he recovered. He was transferred to an intensive care unit (ICU) and, because of some difficulties with airway management, was sedated and intubated; he had two more episodes of bradycardia and resuscitation maneuvers. Electrocardiography revealed ST-segment depression from V4 to V6, and blood cardiac biomarkers rise was observed (tropo$\operatorname{nin}$ I 8,531 pg/mL; creatine kinase 1,800 U/L; myoglobin $625 \mathrm{ng} / \mathrm{mL}$ ). A transthoracic echocardiography revealed left ventricular systolic dysfunction with an estimated ejection fraction of $30 \%$, apical septal hypokinesia/dyskinesia, and a mild mitral regurgitation. He was admitted to our institution and an emergent coronary angiography was performed that showed a distal left main stem stenosis of $95 \%$, proximal left anterior descending (LAD) artery stenosis of $99 \%$, an ostial circumflex artery stenosis of $90 \%$, and an ostial right coronary artery lesion of $99 \%$ (Figure 1).

At heart team discussion, it was decided to perform an emergent coronary artery bypass grafting (CABG) surgery. There was no vein or radial artery considered of good quality for a bypass (atrophied, small dimensions and somewhat deformed limbs associated with some skin alterations with a sclerodermoid change, hypopigmentation, and brightness changes), and so, we used both internal thoracic arteries (ITAs). Both thoracic arteries had diameter of $1.25 \mathrm{~mm}$, and during harvesting of a skeletonized left internal thoracic artery (LITA), there were a couple of periods of hemodynamic instability. Despite the fact that there were no real periods of low cardiac output, early institution of cardiopulmonary bypass was accomplished, in order to avoid hemodynamic deterioration. An arterial cannula was placed in the distal ascending aortic $(16 \mathrm{~F})$, venous cannula into the right atrium $(24 \mathrm{~F})$, and a left decompression vent in the right superior pulmonary vein. The left ITA was completely harvested, and with the beating heart and use of a cardiac stabilizer, an LITA to LAD anastomosis was performed to the 1.5 -mm LAD artery. Still on CPB, the right internal thoracic artery (RITA) was then harvested (skeletonized), and later an anastomosis to an obtuse marginal artery $(1.5 \mathrm{~mm})$ was performed. A " $\mathrm{T}$ ” anastomosis of RITA in LITA was conducted. There was not enough length of RITA to try to reach a right coronary artery collateral, so the right

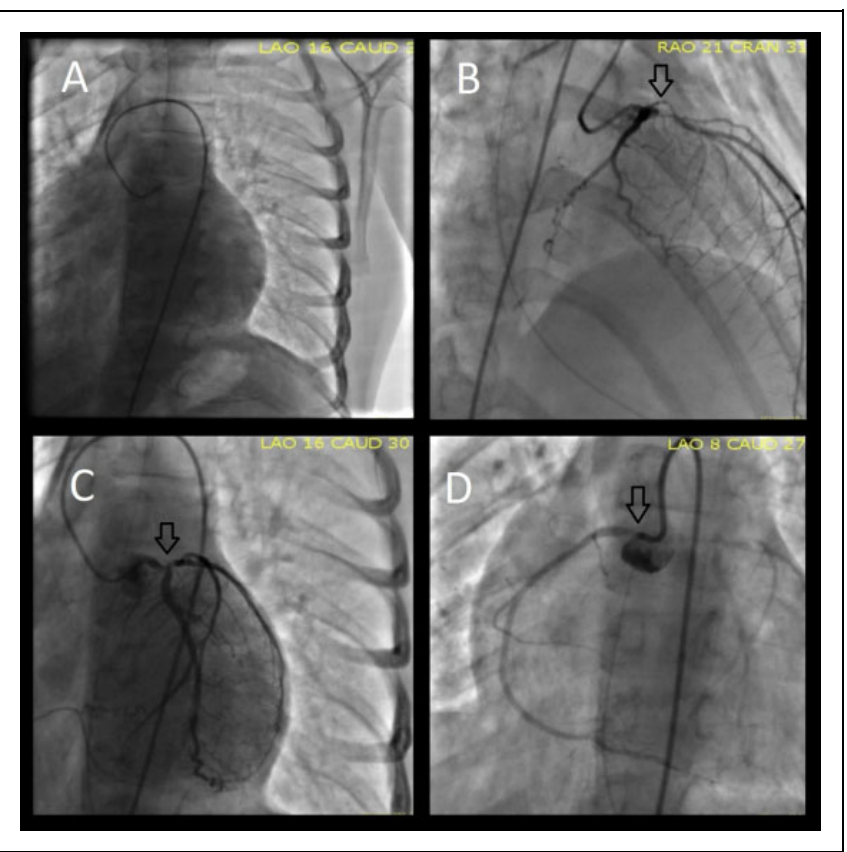

Figure I. Emergent coronariography: A, Overview of thoracic wall and heart; (B) proximal left anterior descending (LAD) stenosis; (C) left main stem and ostial circumflex artery lesion; (D) ostial right coronary artery subtotal occlusion. Note absence of calcifications and almost normal appearance of the distal vessels.

system was not bypassed. During weaning from bypass support, right ventricular dysfunction was present, prompting administration of low doses of milrinone $(0.4 \mu \mathrm{g} / \mathrm{kg} / \mathrm{min})$ and noradrenaline $(0.07 \mu \mathrm{g} / \mathrm{kg} / \mathrm{min})$ with hemodynamic status improvement.

After 10 hours in the ICU, hemodynamic stability was achieved, with progressive discontinuation of all inotropic drugs in 36 hours. When sedation was weaned, he had never recovered consciousness; diabetes insipidus was diagnosed in the second postoperative day with difficult sodium control. Further neurological evaluation was conducted: isoelectric electroencephalogram was recorded and the head computerized tomography disclosed general brain edema and signs of probable vascular stasis. Eventually on fourth postoperative day, brain death was declared and, after discontinuation of invasive therapeutic measures, he was declared death.

\section{Discussion}

Cardiovascular events are the most common cause of death in progeria patients, and so treatment and prevention of these events are paramount. Recent reports describe vascular lesions in these patients not only similar to the aging atherosclerosis (lipid-driven core and chronic inflammatory plaques) but also as having a prominent adventitial fibrosis of the arterial wall. Probably, the evolution of these lesions can precipitate different types of acute cardiovascular events (namely stroke and myocardial infarction), with some reports stating that the conventional therapy can have a role with good clinical results. ${ }^{3,4}$ 
There are no clear indications about early diagnosis of coronary artery disease in this population, but because of the high prevalence, we believe that elective exercise testing or cardiac imaging (eg, computed tomography angiography) should be used do rule out severe coronary artery disease, in order to prevent coronary ischemic events in the future.

Life span of progeria patients is only till second decade of life and so to treat patients who already survived the expected life span with such a severe condition could be debated. But, like in our case, these children have social life and they attend school and/or some other social activities, besides they are integrated in a familiar context, so we believe it is advisable to treat various conditions they develop.

After stressful situations, such as surgical procedures, lifethreatening events can happen. After orthopedic surgery, serious adverse events such as fat or pulmonary embolism, myocardial infarction, or stroke may occur, and they should be considered on the management of cardiac arrest; as so, specific studies should be conducted to rule out these conditions.

In our case, the severity of the coronary disease precluded a percutaneous coronary intervention, and surgery, even in this high-risk setting, was our option.

When cardiac arrest developed and advanced cardiac life support was instituted, our patient was sedated and intubated. Because myocardial infarction was still evolving with hemodynamic instability, reassurance of neurological status previously to our surgery was not considered a priority, regarding the only few minutes he allegedly was with low cardiac output.

"Off-pump CABG" would be our preferred technique to use (because it is the technique we are used in almost all CABG cases, and by trying to avoid the extracorporeal circulation proinflammatory state or the resultant ischemia of an aortic crossclamping), but the hemodynamic instability did not allow it. Because saphenous veins or radial arteries were not available (many conjunctive-osteomuscular abnormalities), the ITAs were the best option. Even though being small, ITAs can be harvested as usual and can be used resulting in optimal anastomosis. We hypothesize that in an elective setting, any CABG technique (on-pump or off-pump) would be feasible. ${ }^{5}$ Another possible conduit that could be used would be the gastroepiploic artery. This conduit is not commonly used and there are not so many centers that still have experience using it. In an emergent scenario is even lesser recommendable because it is not so easy to harvest and it could further complicate the conduction of the surgical procedure.

In this case, we revascularize only the left system that we considered the most important and accept to leave the operating room with an incomplete revascularization. The next days, with recovery of hemodynamic status, as well as the good biventricular function on the postoperative echocardiogram evaluation reassured us of this decision.

Treatment of coronary disease in patients with progeria is very rare. To our knowledge, there is only one report of a coronary percutaneous intervention in a progeria patient, performed in an emergent setting because of an evolving myocardial infarction and with good hemodynamic and clinical result. ${ }^{4}$ Pediatric CABG in progeria patients is also very rare, with only one series of pediatric $\mathrm{CABG}$ reporting experience with one progeria patient. In this publication, it is described the results of 14 surgeries, but only one in a progeria patient, and, as in our case, perioperative death had occurred. The circumstances in which it had happened were not specified. ${ }^{5}$ There are some publications about surgery in syndrome-like forms of progeria but not in the typical HGPS, and so their results cannot be extrapolated. ${ }^{6}$ Pediatric CABG in itself is also a rare procedure, with biggest series having only some dozens patients and most related to Kawasaki disease.

The overall result in our case was not satisfactory mostly because of the acuteness and emergent setting in which the surgery was performed. It is our conviction that the principal brain insult occurred during the cardiac arrest in the pediatric ward and this, associated with the carotid and cerebral artery disease already present, was responsible for the brain death.

Nevertheless, we demonstrate that CABG is feasible in this population, as also recovery from an acute coronary syndrome and low output syndrome is possible with surgical revascularization and optimized perioperative care.

\section{Declaration of Conflicting Interests}

The author(s) declared no potential conflicts of interest with respect to the research, authorship, and/or publication of this article.

\section{Funding}

The author(s) received no financial support for the research, authorship, and/or publication of this article.

\section{ORCID iD}

Rui Pedro Soares Cerejo, MD (D) https://orcid.org/0000-0001-66957866

\section{Supplemental Material}

Supplemental material for this article is available online.

\section{References}

1. Kashyap S, Shanker V, Sharma N. Hutchinson-Gilford progeria syndrome: a rare case report. Indian Dermatol Online J. 2014; 5(4): 478-481.

2. Ilyas S, Ilyas H, Hammed A, Ilyas M. Progeria syndrome with cardiac complications. J Pak Med Assoc. 2013;63(9): 1183-1185.

3. Hisama FK, Lessel D, Leistritz D, et al. Coronary artery disease in a Werner syndrome-like form of progeria characterized by low levels of progerin, a splice variant of lamin A. Am J Med Genet A. 2011;155A(12): 3002-3006.

4. Roggen M, Dubois C, Gewillig M. Coronary artery stenting in a patient with progeria. Catheter Cardiovasc Interv. 2017;90(2): E38-E40.

5. Viola N, Alghamdi A, Al-Radi O, Coles J, Van Arsdell G, Caldarone C. Midterm outcomes of myocardial revascularization in children. J Thorac Cardiovasc Surg. 2010;139(2): 333-338.

6. Hisam F, Lessel D, Leistriz D, et al. Coronary artery disease in a Werner syndrome-like form of progeria characterized by low levels of progerin, a splice variant of lamin A. Am J Med Genet A. 2011; 0(12): 3002-3006. 